\title{
SARS-CoV-2 Omicron variant replication in human respiratory tract ex vivo
}

\section{Michael C. W. Chan ( $\nabla$ mchan@hku.hk)}

The University of Hong Kong

\section{Kenrie PY Hui}

The University of Hong Kong

John Ho

The University of Hong Kong

\section{Man-chun Cheung}

The University of Hong Kong

\section{Ka-chun $\mathbf{N g}$}

The University of Hong Kong

\section{Rachel Ching}

The University of Hong Kong

\section{Ka-ling Lai}

The University of Hong Kong

\section{Tonia Kam}

The University of Hong Kong

\section{Haogao Gu}

The University of Hong Kong https://orcid.org/0000-0002-7541-4262

\section{Ko-Yung Sit}

Queen Mary Hospital

\section{Michael Hsin}

Queen Mary Hospital

\section{Wing-Kuk Au}

University of Hong Kong https://orcid.org/0000-0002-6196-7317

\section{Leo Poon}

University of Hong Kong https://orcid.org/0000-0002-9101-7953

\section{Malik Peiris}

University of Hong Kong https://orcid.org/0000-0001-8217-5995

\section{John Nicholls}

The University of Hong Kong https://orcid.org/0000-0001-7217-7444 


\section{Keywords:}

Posted Date: December 22nd, 2021

DOI: https://doi.org/10.21203/rs.3.rs-1189219/v1

License: (c) (1) This work is licensed under a Creative Commons Attribution 4.0 International License. Read Full License

Version of Record: A version of this preprint was published at Nature on February 1st, 2022. See the published version at https://doi.org/10.1038/s41586-022-04479-6. 


\section{Abstract}

Emergence of SARS-CoV-2 variants of concern (VOC) with progressively increased transmissibility between humans is a threat to global public health. Omicron variant also evades immunity from natural infection or vaccines ${ }^{1}$. It is unclear whether its exceptional transmissibility is due to immune evasion or inherent virological properties.

We compared the replication competence and cellular tropism of the wild type (WT) virus, D614G, Alpha, Beta, Delta and Omicron variants in ex vivo explant cultures of human bronchus and lung. Dependence on TMPRSS2 for infection was also evaluated. We show that Omicron replicated faster than all other SARS-CoV-2 in the bronchus but less efficiently in the lung parenchyma. All VOCs had similar cellular tropism as the WT. Delta was more dependent on serine protease than other VOCs tested.

Our findings demonstrate that Omicron is inherently able to replicate faster than other variants known to date and this likely contributes to its inherently higher transmissibility, irrespective of its ability to evade antibody immunity. The lower replication competence of Omicron in human lung may be compatible with reduced severity but the determinants of severe disease are multifactorial. These findings provide important biological clues to the transmissibility and pathogenesis of SARS-CoV-2 VOCs.

\section{Introduction}

Since the emergence of COVID-19 in December 2019, the causative agent SARS-CoV-2, has continued to evolve in humans, generating variants of progressively increased transmissibility within humans. The World Health Organization (WHO) has classified several lineages as Variant of Concern (VOC) on the grounds of their high transmission rate, potential for immune evasion, unusual epidemiological properties or adverse impact on diagnostics and therapeutics ${ }^{2}$. These VOCs include the B.1.1.7 (Alpha), B.1.351 (Beta), P.1 (Gamma), B.1.617.2 (Delta), and B.1.1.529 (Omicron) virus lineages.

The first virus variant emerged in February 2020 carrying the D614G amino acid substitution in the S protein and this quickly became the dominant virus variant globally. It was shown to replicate more rapidly in primary airway epithelial cells and in the nasal cavity of experimentally infected hamsters, explaining its greater transmissibility in humans ${ }^{3}$. The B.1.1.7 lineage (Alpha variant), first reported in the UK in September 2020, had 13 mutations in the spike (S) protein and the N501Y, P681 H mutations in the spike protein contributed to its increased transmissibility ${ }^{4,5}$. The deletion at 60-70 led to loss of detection of the detection of the $S$ gene target in some diagnostic RT-PCR, since termed S-gene target failure ${ }^{6}$. The B.1.351 lineage (Beta variant) first detected in South Africa in August 2020, contained 10 mutations in the $S$ protein and mutations N501Y and K417N enhanced viral transmission while E484K contributed to immune evasion ${ }^{7,8}$. The B.1.617.2 lineage (Delta variant) was first identified in the state of Maharashtra, India in October 2020, had multiple amino acid substitutions in the S protein including L452R and E484Q which cause enhancement of ACE2 binding, transmission and immune evasion ${ }^{9,10}$. The Omicron variant was identified in Botswana and South African in November 2021 and was designated as VOC by the 
WHO in the same month. It has 37 amino acid substitutions in the S protein, 15 of these being in the receptor binding domain. Thus, it was likely that the phenotype of the virus would be markedly affected in transmission, escape for prior immunity of both. As of 14 December 2021, the Omicron variant has been detected in 76 countries globally ${ }^{11}$. Differences in disease severity between variants has been more subtle. A modest increase in disease severity has been reported with Delta VOC with higher hospital admissions compared with the alpha VOC ${ }^{12,13}$. The Omicron variant contains 37 amino acid substitutions in the spike protein ${ }^{14}$ and these are likely to have major implications on transmissibility, disease severity and immune evasion from serum neutralizing antibodies from both infection-, vaccineelicited and therapeutic monoclonal antibodies. However, there is limited information on the biological or virological characteristics of this variant. Omicron virus replication is ACE-2 dependent and it is able to markedly evade neutralizing antibody elicited by past infection or vaccination 1 .

Correlation of the phenotype of a SARS-CoV-2 variant in vitro with epidemiology is well illustrated by the experimental observations with the D614G mutation. An isogenic wild-type virus engineered to carry the spike D614G substitution had enhanced viral replication in human lung epithelial cells and primary human airway tissues by increasing the infectivity and stability of virions ${ }^{15}$. Hamsters infected with this virus produced higher infectious titres in nasal washes and the trachea, but not in the lungs, supporting clinical evidence showing that the mutation enhances viral loads in the upper respiratory tract of COVID19 patients and may increase transmission. But there are no studies using physiologically relevant experimental models investigating the phenotype of the Omicron variant in comparison with previous variants of SARS-CoV-2.

We have previously used ex vivo explant cultures of the human bronchus and lung parenchyma to study virus replication competence and cellular tropism for avian influenza viruses ${ }^{16,17}$, MERS ${ }^{18-20}$ and SARS$\mathrm{CoV}-2^{21}$. This provided a suitable platform to rapidly compare the replication profile and tropism of the Omicron variant with others and to provide insight into the observed epidemiology of this variant. We initially compared the virus replication kinetics of wild type SARS-CoV-2 (WT) with the D614G variant and VOCs from B.1.1.7 (Alpha) and B.1.351 (Beta) lineages in ex vivo explant cultures of human bronchus and lung. In a subsequent set of experiments, we compared viral replication profiles of WT with B.1.617.2 (Delta) and B.1.1.529 (Omicron) lineages. We also investigated the virus tropism and innate immune responses elicited by these variants. Finally, we compared the dependency of WT, Delta and Omicron variants on TMPRSS2 for their replication.

\section{Results}

\section{Higher replication of Omicron in bronchus}

We first compared the replication kinetics of WT strain, D614G, Alpha and Beta virus strains isolated from returning travellers or the community in Hong Kong in ex vivo cultures of human bronchus and lung by titrating infectious virus using $50 \%$ tissue culture infectious dose $\left(\mathrm{TCID}_{50}\right)$ titrations. The only significant difference observed was a higher replication Beta variant in the human bronchus at $72 \mathrm{hpi}$; no significant 
difference being noted between viruses at 24 and $48 \mathrm{hpi}$ (Figure 1a). When WT, Delta and Omicron variants were compared, Omicron variant replicated to significantly higher titres than WT or Delta at 24 and $48 \mathrm{hpi}$; the difference being over 70-fold (Figure 1b). At $72 \mathrm{hpi}$, both Delta and Omicron viruses replicated significantly more than WT in human bronchus but there was no significant difference between Delta and Omicron viruses. When these experiments were done at $33^{\circ} \mathrm{C}$, the viral titres were similar to those at $37^{\circ} \mathrm{C}$ for each virus (data not shown). In ex vivo cultures of human lung, the only significant difference observed was reduced replication of Omicron compared to WT at 24, 48 and $72 \mathrm{hpi}$ (Figure $1 \mathrm{c}, \mathrm{d})$. These findings in bronchus and lung were confirmed in area-under-curve (AUC) analysis of aggregate virus titres at 24-72 hpi (Figure 1e,f).

The tropism of each virus variant in the bronchus and lung was visualized with immunohistochemical staining for SARS-CoV-2 nucleoprotein (Figure 2). The virus variants did not appear to differ in cell tropism in the bronchus. In bronchus tissues infected ex vivo, Omicron had evidence of extensive virus infection with ciliated epithelium, goblet cells and club cells showing infection in WT, Alpha, Beta, Delta and Omicron viruses by using immunofluorescent staining (Figure $2 a$ and 3 ). The virus variants did not appear to differ in cell tropism in the lung (Figure 2b). Immunohistochemical staining showed positive staining in the lung tissues with the spindle shaped antigen positive cells morphologically resembling type 1 pneumocytes. In tissues infected with the omicron variant there were decreased numbers of antigen positive cells identified.

\section{ACE2 and TMPRSS2 expression in respiratory tract}

Entry of SARS-CoV-2 into cells requires both angiotensin-converting enzyme 2 (ACE2) and transmembrane serine protease 2 (TMPRSS2) which cleaves and activates the spike at the S1-S2 junction $^{22}$. ACE-2 expressed at cell surface exists as short and long forms, with the short form lacking the domain that binds to SARS-CoV-2 spike ${ }^{23}$. We showed that there was significantly higher expression of both long and short form ACE2 in human bronchus than in the lung (Figure 4a). Immunohistochemical staining also showed clear evidence of more extensive ACE2 staining in bronchus than lung (Figure 4b and 4c). We next investigated the TMPRSS2 expression and showed significantly higher expression of TMPRSS2 in bronchus than lung by mRNA expression levels (Figure 4a).

\section{Variants and dependence of TMPRSS2}

We investigated WT, Delta and Omicron virus replication for their dependence on TMPRSS2. We compared the viral replication kinetics of wild-type, Delta and Omicron in Vero E6 and Vero E6-TMPRSS2 $(\mathrm{E} 6 / \mathrm{T} 2)$ cells. While virus titres at $24 \mathrm{hpi}$ was higher in E6/T2 cells in all three viruses, the magnitude of the difference was 100-1000 fold in Delta, 5-6 fold for Omicron and marginal for WT (Figure 4c). The AUC levels of the viral titres at 24-48 hours confirmed these findings (Figure 4d). We next infected Vero E6/T2 
cells with WT, Delta and Omicron in the presence of a serine protease inhibitor camostat mesylate which blocks TMPRSS2 activities ${ }^{22}$. Delta and Omicron variants were propagated in Vero E6/T2, in order to exclude selection of TMPRSS2-dependent virus during propagation, Beta which was used as a control virus was also propagated in Vero E6/T2 cells. The viability of Vero E6/T2 was above $80 \%$ when treated with camostat mesylate at concentrations up to $300 \mu \mathrm{M}$ (Extended Data Figure 1). In the presence of camostat mesylate treatment, only Delta was sensitive to the inhibitor and the half-maximal inhibitory concentration (IC50) was $10.66 \mu \mathrm{M}$ (Figure 4f). In contrast, the IC ${ }_{50}$ of WT, Beta and Omicron was higher than $300 \mu \mathrm{M}$.

\section{Discussion}

Preliminary epidemiological data from the United Kingdom suggests that the risk of household transmission with Omicron is 3.2 (95\% confidence interval 2.0-5.0) higher than with Delta, which in turn was a highly transmissible virus, and that Omicron has a Reproduction number $(R)$ of $3.7^{24}$. It has been unclear whether this increased efficiency of transmission is driven by mutations that confer immune escape from antibody in the population elicited by prior infection or vaccination, by intrinsic viral factors or a combination of the two. It is increasingly clear that high neutralizing antibody titres elicited by prior infection or immunization are markedly compromised by Omicron virus ${ }^{1}$. Our results suggest that the Omicron variant has a substantial (over 70-fold increase) and significantly higher replication competence in the human bronchus compared to both wild-type and Delta viruses at $24 \mathrm{hpi}$. Higher infectious virus load in conducting airways is likely to result in increased amounts of infectious virus released while breathing or speaking thus enhancing transmission by the airborne route. Examples of such airborne transmission of Omicron have been documented ${ }^{14}$. Infectious SARS-CoV-2 has been detected in fine aerosol particles in air exhaled by patients with COVID-1925. Thus, our data indicates that Omicron has intrinsic biological properties that potential mediate enhanced transmission, which may also synergise with its ability for evading prior immunity in leading to enhanced overall transmissibility in the community. The mechanistic reasons underlying this increased replication competence remains to be elucidated. The Omicorn variant has 37 amino acid substitutions in the spike protein, 15 of which are in the receptor binding domain ${ }^{14}$. Infection with Omicron is ACE-2 dependent ${ }^{1}$ and that the binding of the spike of Omicron spike to ACE-2 is enhanced when compared to the wild-type virus ${ }^{26}$. We showed that there is more extensive ACE2 expression in human bronchus than lung which may explain the enhanced replication SARS-CoV-2 in the bronchus. Omicron also has amino acid substitutions in the nucleocapsid protein (R203K and G204R) that have been associated with enhanced virus replication ${ }^{27}$.

Epidemiological studies suggested that Delta variant was substantially more transmissible than Alpha variant ${ }^{28}$, which was itself was more transmissible than the earlier virus stains ${ }^{4}$. Thus, our data indicating that Delta variant has higher titres of infectious virus than wild-type virus in the bronchus is also compatible with the epidemiological observations. It has been shown that pseudoviruses expressing Delta spike are better able to infect ACE2 ${ }^{\text {low }}$ human bronchial epithelial cells compared to previous variants ${ }^{29}$. Moreover, the spike of Delta is in a predominantly cleaved state which may promote its 
replication efficiency in human airway ${ }^{30}$. P681R enhanced the cleavage of furin-cleavage sequences, which attributes to the enhanced fitness of Delta over Alpha in a competition assay in Calu-3 and human airway epithelium in vitro models ${ }^{31,32}$.

Our data showing that the Omicron variant had lower viral replication competence in the lung, compared to the bronchus is of particular interest. This difference in virus tropism is also confirmed by the immunohistochemistry studies showing less virus infected cells in human lung explant cultures ex vivo. The biological determinants of this divergence in comparative replication competence of Omicron and Delta variants in the bronchus and lung remains to be explored. These observations may suggest that Omicron may have reduced clinical severity but such interpretations need to be qualified because the disease severity of COVID-19 is determined not only by virus replication but also by dysregulated innate immune responses. Reliable epidemiological assessments of severity of disease caused by Omicron is still awaited. Although reports from South Africa suggest that hospitalization and intensive care admissions appears to be less common during the recent Omicron outbreak ${ }^{33}$, preliminary assessment of hospitalisation and asymptomatic infection indicators in the UK did not show differences in hospitalization rates with Delta and Omicron variants ${ }^{34}$.

Our finding that Omicron is less dependent on TMPRSS2 activation compared with Delta suggests that Omicron may have a wider spectrum of target cells comparing to Delta and also that the use of therapeutic inhibitors of TMPRSS2 may be of limited benefit in the management of clinical infections with the Omicron variant. Though there have been single cell studies on the distribution of TMPRSS 2 in cell cultures, the extent of distribution is hampered by sensitivity and specificity of antibodies in fixed tissues.

One of the limitations of this study is that only one virus strain from each lineage was tested. Furthermore, all six virus variants were not tested in parallel in the same experiment, but this is very difficult to carry out logistically because of limited tissue available to test six viruses with replicates.

In summary, our findings reveal faster and enhanced viral replication efficiency of Omicron in the human bronchus compared with the precedent lineages, suggesting an intrinsic capacity for enhanced transmission which is compatible with current epidemiological data. The lower replication competence of Omicron in human lung suggests that it may cause less severe COVID-19 than Delta. Even if disease severity is modestly reduced, the very efficient transmissibility of Omicron will pose a major threat to global public health and health care systems. Investigations on preventing Omicron infection via booster vaccination booster and therapeutic options are urgently needed.

\section{Declarations}

\section{Acknowledgements}

Hin-Wo Yeung, Cassia Lin (School of Public Health, LKS Faculty of Medicine, The University of Hong Kong, Hong Kong SAR, China) and Kevin Fung (Department of Pathology, LKS Faculty of Medicine, The 
University of Hong Kong, Hong Kong SAR, China) provided technical support.

We acknowledge research funding from the National Institute of Allergy and Infectious Diseases, National Institutes of Health, Department of Health and Human Services (Contract No. 75N93021C00016) and the Theme-Based Research Scheme (Ref: T11-705/21N and T11-712/19-N) under University Grants Committee of Hong Kong Special Administrative Region. The funding sources had no roles in the writing of the manuscript or the decision to submit it for publication. The authors have not been paid to write this article by a pharmaceutical company or other agency. The authors had full access to all the data in the study and had final responsibility for the decision to submit for publication. All data generated or analysed during this study are included in this published article (and its supplementary information files).

\section{Authors' contributions}

KPYH: study design, coordination, analysis, interpretation of results, perform experiments and writing of the manuscript; JCWH, MCC, KCN, RHHC, KLL, TTK: perform experiments, analysis and interpretation of results; HG: perform analysis and interpretation of sequencing results; KYS, MKYH, TWKA: provide human lung and bronchus tissue and critical review of the manuscript; LLMP: analysis and interpretation of sequencing results and critical review of the manuscript; MP: analysis and interpretation of results, writing and revision of the manuscript; JMN: analysis, co-ordination of sample collection, and interpretation of results from immunohistochemical staining and critical review of the manuscript; MCWC: study design, overall coordination, interpretation of results and writing of the manuscript.

\section{Conflict of interest}

There is no conflict of interest for all authors.

\section{Role of the funding source}

The sponsors had no role in study design, data collection, analysis, or interpretation of the data, or in the writing of the report. The corresponding author had full access to all of the data and the final responsibility to submit for publication.

\section{Methods}

\section{SARS-CoV-2 isolation}


Vero E6 cells were used for virus isolation and propagation of wild-type virus, D614G strain and Alpha variant, and Vero E6-TMPRSS2 overexpressed cells ${ }^{35}$ were used for Beta, Delta and Omicron variant. Both cell-lines were cultured in DMEM with 10\% FBS. The original clinical specimens were collected from SARS-CoV-2 confirmed patients in Hong Kong from January 2020 to November 2021 (Extended Data Table 1) and isolated as previously described ${ }^{36}$. The virus stock was aliquoted and stored frozen at $-80^{\circ} \mathrm{C}$. Aliquots were titrated to determine $50 \%$ tissue culture infection dose $\left(\mathrm{TCID}_{50}\right)$ in respective cell lines. The experiments were carried out in a Bio-safety level 3 (BSL-3) facility at the School of Public Health, LKS Faculty of Medicine, The University of Hong Kong. Informed consent was obtained from all subjects and approval was granted by the Institutional Review Board (IRB) of the University of Hong Kong and the Hospital Authority (Hong Kong West) (IRB approval no: UW 20-167 and UW19-802).

\section{Ex vivo cultures and infection of human respiratory tract}

Fresh non-tumour bronchus $(n=12)$ and lung $(n=12)$ tissues were obtained from patients with age 51-78 years undergoing elective surgery in Department of Surgery at Queen Mary Hospital (Pok Fu Lam, Hong Kong, China) from May to December 2021 and were removed as part of routine clinical care but surplus for routine diagnostic requirements as detailed previously. ${ }^{37,38}$ Sampling of tissues was defined by obtaining informed consent and convenience. The donor information was listed in the Extended Data Table 2. The virus infection procedures were performed as previously described ${ }^{36}$. Briefly, pieces of human bronchus and lung tissues were infected with each virus at $5 \times 10^{5} \mathrm{TCID}_{50} / \mathrm{ml}$ for $1 \mathrm{~h}$ at $37^{\circ} \mathrm{C}$. Each tissue fragment was washed three times in culture medium to remove residual virus inoculum, topped up with fresh medium and incubated at $37^{\circ} \mathrm{C}$ as indicated. Mock-infected tissues served as negative controls. Aliquots of culture medium were removed at times indicated and stored at $-80^{\circ} \mathrm{C}$ until titration. Infectious viral titres in culture supernatants were assayed by $\mathrm{TCID}_{50}$ in Vero E6 or Vero E6-TMPRSS2 cells respectively, depending on the cells used for virus isolation and passage. Infected tissues were fixed in $10 \%$ formalin and processed for immuno-staining at 72 hours post infection.

\section{Viral titration by $\mathrm{TCID}_{50}$ assay}

A confluent 96-well tissue culture plates of Vero-E6 or Vero E6-TMPRSS2 cells was prepared one day before the virus titration $\left(\mathrm{TCID}_{50}\right)$ assay. Cells were washed once with PBS and replenished with DMEM (Gibco) with $2 \%$ foetal bovine serum (Gibco) supplemented with $100 \mathrm{units} / \mathrm{ml}$ penicillin and $100 \mu \mathrm{g} / \mathrm{ml}$ streptomycin (Gibco). Serial dilutions of virus supernatant, from $0.5 \mathrm{log}$ to $7 \mathrm{log}$, were performed and each virus dilution added to the plates in quadruplicate. The plates were observed for cytopathic effect daily. The end-point of viral dilution leading to CPE in $50 \%$ of inoculated wells was estimated using the Karber method ${ }^{39}$. Area under the curve (AUC) was calculated from the viral titers from different time points indicated in the $\mathrm{Y}$-axis. 


\section{Immuno-staining of paraffin-embedded tissues}

Human respiratory tract tissues (lung and bronchus) were fixed with $10 \%$ formalin overnight at $4{ }^{\circ} \mathrm{C}$ and the fixed tissues were embedded in paraffin blocks. For immunohistochemistry, the $4 \mu \mathrm{m}$ sliced sections were microwaved for $15 \mathrm{~min}$ for antigen retrieval. Endogenous peroxidase activity was stopped by quenching the tissue sections with $3 \% \mathrm{H}_{2} \mathrm{O}_{2}$ for $20 \mathrm{~min}$. The slides were then blocked with $10 \%$ normal horse serum at room temperature (RT) and incubated with primary antibodies (SARS-CoV-2 Nucleoprotein (NP) (Sino Biological) or ACE2 (abcam) antibodies for 90 min at RT followed by peroxidase (HRP)conjugated anti-rabbit antibody (Vector Laboratory). The sections were developed using NovaRED Substrate Kit (Vector Laboratory). The cell nuclei were counterstained with Mayer's Hematoxylin.

To characterize the SARS-CoV-2-infected cells, double-antibody immunofluorescence staining of SARSCoV-2 NP antibody with different cellular markers was performed. The tissue sections were first stained with SARS-CoV-2 NP antibody similarly as mentioned above, except after SARS-CoV-2 NP antibody incubation, the sections were incubated with alkaline phosphatase conjugated anti-rabbit or anti-mouse antibody (Vector Laboratory) and developed using Vector ${ }^{\circledR}$ Red (VR) Substrate Kit (Vector Laboratory). The sections were then microwaved, incubated with SCGB1A1/CC10 (Protein-tech), acetylated a Tubulin (Santa Cruz), MUC5AC (Thermo-Fisher), for $90 \mathrm{~min}$ at room temperature followed by goat anti rabbitAF488 or goat anti mouse-AF488. The cell nuclei were counterstained with DAPI (blue). The sections were imaged using Nikon Eclipse Ti-S microscope.

\section{Real-time PCR assay}

RNA was extracted from homogenized tissues at $72 \mathrm{~h}$ post infection (hpi) using RNeasy Micro Kit (Qiagen). The viral RNA in culture supernatants were extracted at $48 \mathrm{hpi}$ using QIAamp Viral RNA Mini Kit (Qiagen). RNA was reverse-transcribed by using random 6-mer primers with PrimeScript RT reagent Kit (Takara). mRNA expression of target genes was detected with the corresponding primers (Extended Data Table 3) using an ABI ViiA 7 real-time PCR system (Applied Biosystems). All procedures were performed according to the manufacturers' instructions. The viral gene and gene expression profiles of cell lysates were quantified and normalized with b-actin as previously described ${ }^{40-43}$.

\section{Replication of SARS-CoV-2 on Vero-E6 and Vero-E6-TMPRSS2 and camostat mesylate treatment}

Vero-E6 and Vero-E6-TMPRSS2 cells in 48-well plates were infected with SARS-CoV-2 at a multiplicity of infection (MOI) of 0.1 for viral replication kinetics. Viral titers in culture supernatants (1-72 hpi) were determined using $\mathrm{TCID}_{50}$ assay (see above). To assess impact of TMPRSS2 inhibitors, the Vero-E6TMPRSS2 cells were infected with SARS-CoV-2 at a MOI of 0.05. Camostat mesylate (Sigma), 0.41- 
$300 \mu \mathrm{M}$, was added $1 \mathrm{~h}$ before, during and after infection. Vehicle was used as a negative control. Antiviral activities were evaluated by quantification of SARS-CoV-2 ORF1b copy number in the culture supernatant by using qPCR at $48 \mathrm{hpi}^{43}$.

\section{Quantification of cell viability}

Cell viability of Vero-E6-TMPRSS2 following the treatment of camostat mesylate was evaluated using Cell Counting Kit 8 (WST-8 / CCK8) (Abcam). In brief, cells grown to 70\% confluency in 48-well plates were incubated for $48 \mathrm{~h}$ in the absence or presence of various concentrations $(0.41-300 \mu \mathrm{M})$ of camostat mesylate. Then, the culture medium was aspirated and washed once with PBS, incubated with CCK8 working solution (CCK8: medium $=1: 10$ ) at $37^{\circ} \mathrm{C}, 5 \% \mathrm{CO}_{2}$ for $2 \mathrm{~h}$. Subsequently, OD values at $450 \mathrm{~nm}$ were measured by a multiplate reader (BMG FLUOstar OPTIMA). Cell viability was expressed as a percentage of that of the control cells.

\section{Statistical analysis}

Experiments with the human ex vivo cultures were performed independently with six different donors. Results shown in figures are geometric mean (+/-SD). Area-under-curve (AUC) was calculated by integrating infectious virus titres at 24-72 hpi in ex vivo bronchus, lung tissues or at 24-48 hpi in cell lines. The differences in log10-transformed viral titres and quantitative cytokine and chemokine mRNA between viruses and over time were compared using two-way ANOVA followed by a Tukey's multiple-comparison test using GraphPad Prism version 9.0.0. Comparison of AUC and quantitative cytokine and chemokine mRNA between viruses were calculated using one-way ANOVA followed by a Tukey's multiple-comparison test. Differences were considered significant at a $p$ value less than 0.05 .

\section{References}

1 Cele, S. et al. SARS-CoV-2 Omicron has extensive but incomplete escape of Pfizer BNT162b2 elicited neutralization and requires ACE2 for infection. medRxiv : the preprint server for health sciences, doi:10.1101/2021.12.08.21267417 (2021).

2 WHO. Tracking SARS-CoV-2 variants, <https://www.who.int/en/activities/tracking-SARS-CoV-2variants/> (2021).

3 Baric, R. S. Emergence of a Highly Fit SARS-CoV-2 Variant. The New England journal of medicine 383, 2684-2686, doi:10.1056/NEJMcibr2032888 (2020).

4 Davies, N. G. et al. Estimated transmissibility and impact of SARS-CoV-2 lineage B.1.1.7 in England. Science (New York, N.Y.) 372, doi:10.1126/science.abg3055 (2021). 
5 Lubinski, B., Tang, T., Daniel, S., Jaimes, J. A. \& Whittaker, G. R. Functional evaluation of proteolytic activation for the SARS-CoV-2 variant B.1.1.7: role of the $\mathrm{P} 681 \mathrm{H}$ mutation. bioRxiv : the preprint server for biology, doi:10.1101/2021.04.06.438731 (2021).

6 Srivastava, S., Banu, S., Singh, P., Sowpati, D. T. \& Mishra, R. K. SARS-CoV-2 genomics: An Indian perspective on sequencing viral variants. Journal of biosciences 46, doi:10.1007/s12038-021-00145-7 (2021).

7 Zhou, D. et al. Evidence of escape of SARS-CoV-2 variant B.1.351 from natural and vaccineinduced sera. Cell 184, 2348-2361.e2346, doi:10.1016/j.cell.2021.02.037 (2021).

8 Khan, A. et al. Higher infectivity of the SARS-CoV-2 new variants is associated with K417N/T, E484K, and N501Y mutants: An insight from structural data. Journal of cellular physiology 236, 70457057, doi:10.1002/jcp.30367 (2021).

9 Ozono, S. et al. SARS-CoV-2 D614G spike mutation increases entry efficiency with enhanced ACE2-binding affinity. Nature communications 12, 848, doi:10.1038/s41467-021-21118-2 (2021).

10 Augusto, G. et al. In vitro data suggest that Indian delta variant B.1.617 of SARS-CoV-2 escapes neutralization by both receptor affinity and immune evasion. Allergy, doi:10.1111/all.15065 (2021).

11 WHO. Weekly epidemiological update on COVID-19 - 14 December 2021. (2021).

12 Sheikh, A., McMenamin, J., Taylor, B. \& Robertson, C. SARS-CoV-2 Delta VOC in Scotland: demographics, risk of hospital admission, and vaccine effectiveness. Lancet (London, England) 397, 2461-2462, doi:10.1016/s0140-6736(21)01358-1 (2021).

13 Twohig, K. A. et al. Hospital admission and emergency care attendance risk for SARS-CoV-2 delta (B.1.617.2) compared with alpha (B.1.1.7) variants of concern: a cohort study. The Lancet. Infectious diseases, doi:10.1016/s1473-3099(21)00475-8 (2021).

$14 \mathrm{Gu}, \mathrm{H}$. et al. Probable Transmission of SARS-CoV-2 Omicron Variant in Quarantine Hotel, Hong Kong, China, November 2021. Emerging infectious diseases 28, doi:10.3201/eid2802.212422 (2021).

15 Plante, J. A. et al. Spike mutation D614G alters SARS-CoV-2 fitness. Nature 592, 116-121, doi:10.1038/s41586-020-2895-3 (2021).

16 Hui, K. P. et al. Tropism and innate host responses of influenza A/H5N6 virus: an analysis of ex vivo and in vitro cultures of the human respiratory tract. The European respiratory journal 49, doi:10.1183/13993003.01710-2016 (2017).

17 Chan, M. C. et al. Tropism and innate host responses of a novel avian influenza A H7N9 virus: an analysis of ex-vivo and in-vitro cultures of the human respiratory tract. The Lancet. Respiratory medicine 1, 534-542, doi:10.1016/s2213-2600(13)70138-3 (2013). 
18 Chan, R. W. et al. Tropism and replication of Middle East respiratory syndrome coronavirus from dromedary camels in the human respiratory tract: an in-vitro and ex-vivo study. The Lancet. Respiratory medicine 2, 813-822, doi:10.1016/s2213-2600(14)70158-4 (2014).

19 Zhou, Z. et al. Phenotypic and genetic characterization of MERS coronaviruses from Africa to understand their zoonotic potential. Proceedings of the National Academy of Sciences of the United States of America 118, doi:10.1073/pnas.2103984118 (2021).

20 Chu, D. K. W. et al. MERS coronaviruses from camels in Africa exhibit region-dependent genetic diversity. Proceedings of the National Academy of Sciences of the United States of America 115, 31443149, doi:10.1073/pnas.1718769115 (2018).

21 Hui, K. P. Y. et al. Tropism, replication competence, and innate immune responses of the coronavirus SARS-CoV-2 in human respiratory tract and conjunctiva: an analysis in ex-vivo and in-vitro cultures. The Lancet. Respiratory medicine, doi:10.1016/s2213-2600(20)30193-4 (2020).

22 Hoffmann, M. et al. SARS-CoV-2 Cell Entry Depends on ACE2 and TMPRSS2 and Is Blocked by a Clinically Proven Protease Inhibitor. Cell, doi:10.1016/j.cell.2020.02.052 (2020).

23 Blume, C. et al. A novel ACE2 isoform is expressed in human respiratory epithelia and is upregulated in response to interferons and RNA respiratory virus infection. Nature genetics 53 , 205-214, doi:10.1038/s41588-020-00759-x (2021).

24 Agency, U. H. S. SARS-CoV-2 variants of concern and variants under investigation (2021).

25 Adenaiye, O. O. et al. Infectious SARS-CoV-2 in Exhaled Aerosols and Efficacy of Masks During Early Mild Infection. Clinical infectious diseases : an official publication of the Infectious Diseases Society of America, doi:10.1093/cid/ciab797 (2021).

26 Cameroni, E. et al. Broadly neutralizing antibodies overcome SARS-CoV-2 Omicron antigenic shift. 2021.2012.2012.472269, doi:10.1101/2021.12.12.472269 \%J bioRxiv (2021).

$27 \mathrm{Wu}, \mathrm{H}$. et al. Nucleocapsid mutations R203K/G204R increase the infectivity, fitness, and virulence of SARS-CoV-2. Cell host \& microbe 29, 1788-1801.e1786, doi:10.1016/j.chom.2021.11.005 (2021).

28 Allen, H. et al. Household transmission of COVID-19 cases associated with SARS-CoV-2 delta variant (B.1.617.2): national case-control study. The Lancet regional health. Europe 12, 100252, doi:10.1016/j.lanepe.2021.100252 (2022).

29 Li, H., Liu, T., Wang, L., Wang, M. \& Wang, S. SARS-CoV-2 Delta variant infects ACE2(low) primary human bronchial epithelial cells more efficiently than other variants. Journal of medical virology, doi:10.1002/jmv.27372 (2021). 
30 Mlcochova, P. et al. SARS-CoV-2 B.1.617.2 Delta variant replication and immune evasion. Nature 599, 114-119, doi:10.1038/s41586-021-03944-y (2021).

31 Liu, Y. et al. Delta spike P681R mutation enhances SARS-CoV-2 fitness over Alpha variant. bioRxiv : the preprint server for biology, doi:10.1101/2021.08.12.456173 (2021).

32 Lubinski, B. et al. Spike protein cleavage-activation mediated by the SARS-CoV-2 P681R mutation: a case-study from its first appearance in variant of interest (VOI) A.23.1 identified in Uganda. bioRxiv : the preprint server for biology, doi:10.1101/2021.06.30.450632 (2021).

33 Dyer, O. Covid-19: Omicron is causing more infections but fewer hospital admissions than delta, South African data show. BMJ (Clinical research ed.) 375, n3104, doi:10.1136/bmj.n3104 (2021).

34 Ferguson N, G. A., Cori A, Hogan A, Hinsley W, Volz E on behalf of the Imperial College COVID-19 response team. Report 49 - Growth, population distribution and immune escape of Omicron in England. . (2021).

35 Matsuyama, S. et al. Enhanced isolation of SARS-CoV-2 by TMPRSS2-expressing cells. Proceedings of the National Academy of Sciences of the United States of America 117, 7001-7003, doi:10.1073/pnas.2002589117 (2020).

36 Hui, K. P. Y. et al. Tropism, replication competence, and innate immune responses of the coronavirus SARS-CoV-2 in human respiratory tract and conjunctiva: an analysis in ex-vivo and in-vitro cultures. The Lancet. Respiratory medicine 8, 687-695, doi:10.1016/s2213-2600(20)30193-4 (2020).

37 Chan, M. C. et al. Tropism and innate host responses of the 2009 pandemic H1N1 influenza virus in ex vivo and in vitro cultures of human conjunctiva and respiratory tract. The American journal of pathology 176, 1828-1840, doi:10.2353/ajpath.2010.091087 (2010).

38 Hui, K. P. et al. Tropism and innate host responses of influenza A/H5N6 virus: an analysis of ex vivo and in vitro cultures of the human respiratory tract. The European respiratory journal 49, doi:10.1183/13993003.01710-2016 (2017).

39 Kärber, G. Beitrag zur kollektiven Behandlung pharmakologischer Reihenversuche. NaunynSchmiedebergs Archiv für experimentelle Pathologie und Pharmakologie 162, 480-483, doi:10.1007/BF01863914 (1931).

40 Chan, R. W. et al. DAS181 inhibits H5N1 influenza virus infection of human lung tissues. Antimicrob Agents Chemother 53, 3935-3941, doi:AAC.00389-09 [pii]10.1128/AAC.00389-09 (2009).

41 Hui, K. P. et al. H5N1 influenza virus-induced mediators upregulate RIG-I in uninfected cells by paracrine effects contributing to amplified cytokine cascades. The Journal of infectious diseases 204, 1866-1878, doi:10.1093/infdis/jir665 (2011). 

influenza A virus (H5N1) is selectively regulated by IFN regulatory factor 3 and p38 MAPK. J Immunol 182, 1088-1098, doi:10.4049/jimmunol.182.2.1088 (2009).

43 Bui, C. H. T. et al. Tropism of SARS-CoV-2, SARS-CoV, and Influenza Virus in Canine Tissue Explants. J Infect Dis 224, 821-830, doi:10.1093/infdis/jiab002 (2021).

\section{Figures}

a

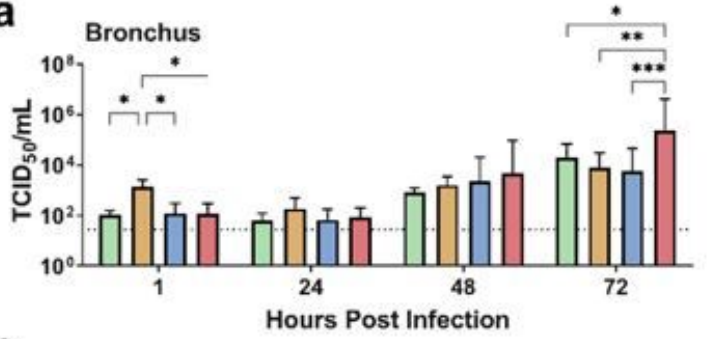

C

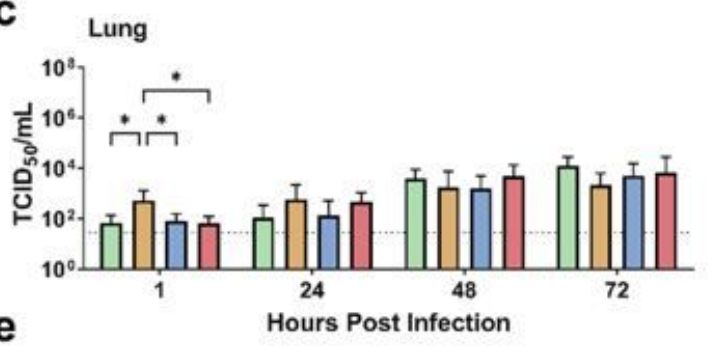

Bronchus

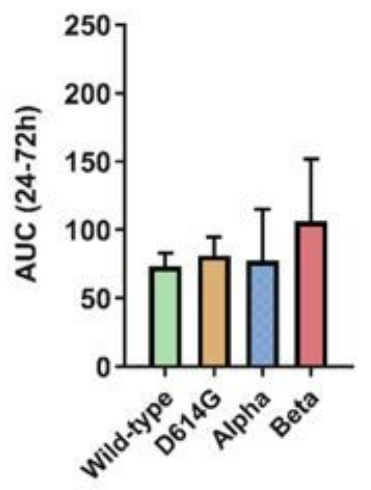

Lung

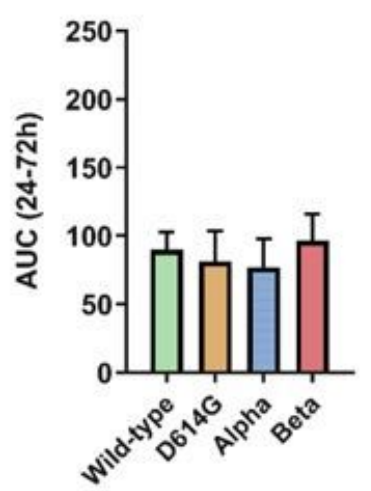

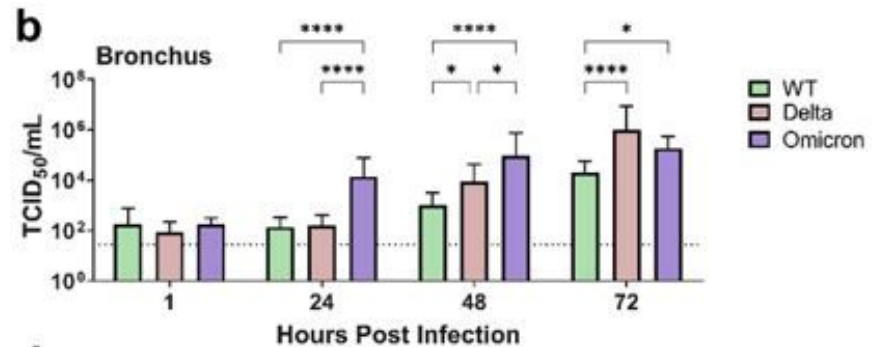

d

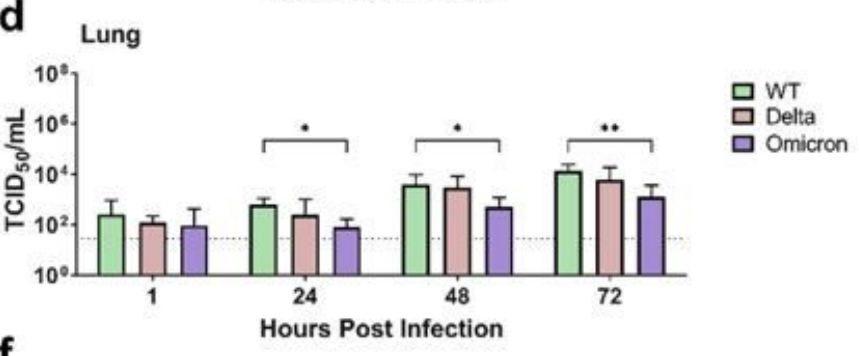

$\mathbf{f}$

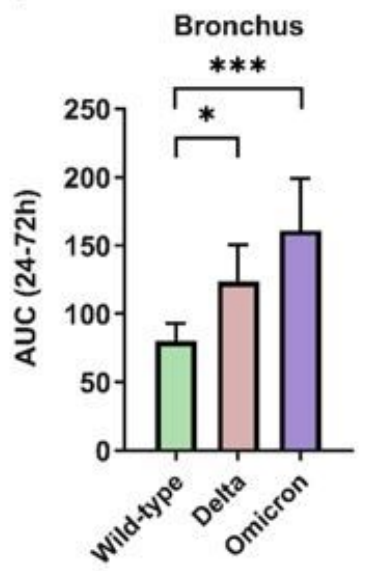

Lung

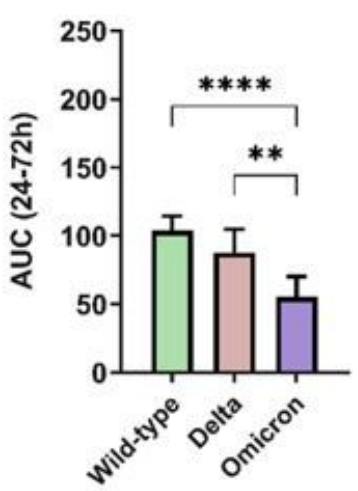

Figure 1

Viral replication kinetics of SARS-CoV-2 variants in ex vivo cultures of human respiratory tract. Human ex vivo cultures of bronchus and lung were infected with $5 \times 10^{5} \mathrm{TCID}_{50} / \mathrm{mL}$ at $37^{\circ} \mathrm{C}$. Virus released in the culture supernatants were measured over time by $\operatorname{TCID}_{50}$ assay. a, c. Viral replication kinetics of wild-type (WT), D614G, Alpha and Beta in human ex vivo cultures of bronchus and lung. b, d. Viral replication kinetics of wild-type (WT), Delta and Omicron in human ex vivo cultures of bronchus and lung. The 
horizontal dotted line denotes the limit of detection in the $\mathrm{TCID}_{50}$ assay. Bar-charts show the geometric mean $(n=6)(S D)$. Statistics were performed using Two-way ANOVA followed by a Tukey's multiplecomparison test. ${ }^{*} p<0.05,{ }^{*} p<0.01,{ }^{* \star *} p<0.001,{ }^{* \star * \star} p<0.0001$. e, f. Viral titers from a to $d$ are depicted as area under the curve (AUC). Bar-charts show the geometric mean $(n=6)(S D)$. Statistics were performed using One-way ANOVA followed by a Tukey's multiple-comparison test. ${ }^{*} p<0.05,{ }^{* \star} p<0.01,{ }^{\star \star \star} p<0.001$, $\star \star \star \star x<0.0001$.

a

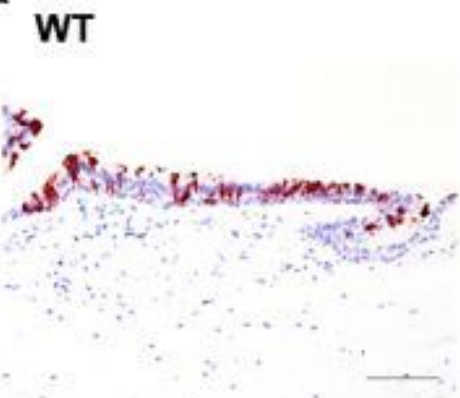

D614G

Alpha

Beta

Delta
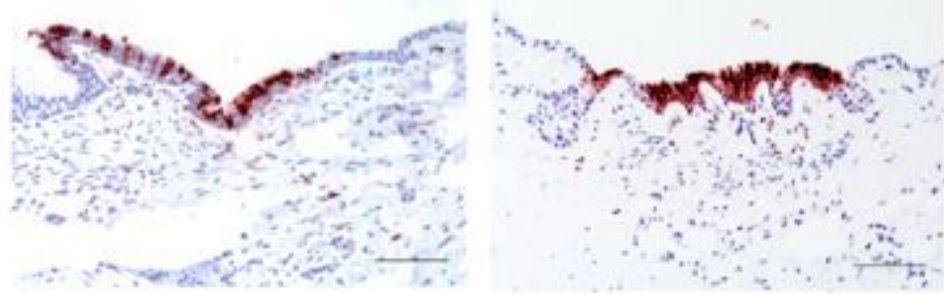

b WT

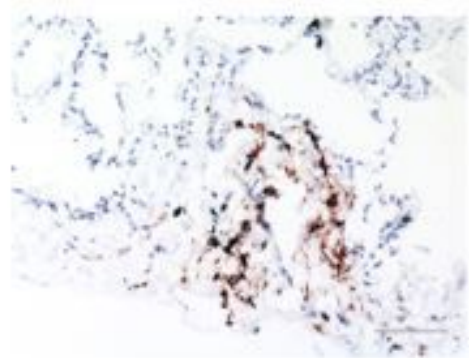

Beta

\section{D614G}

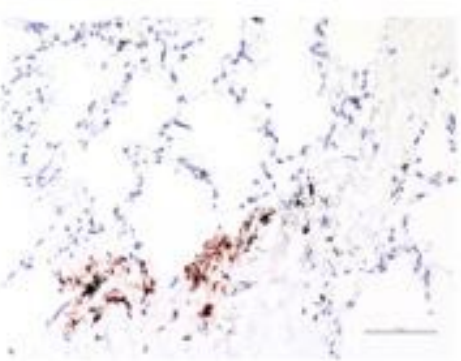

\section{Delta}

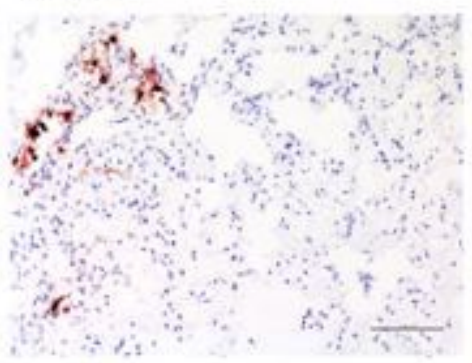

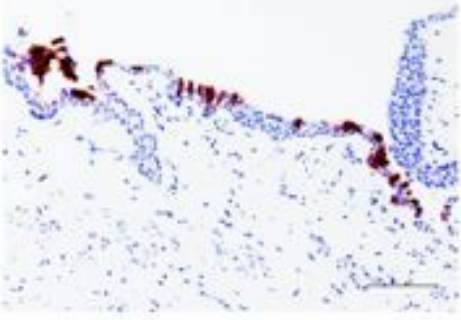

Omicron

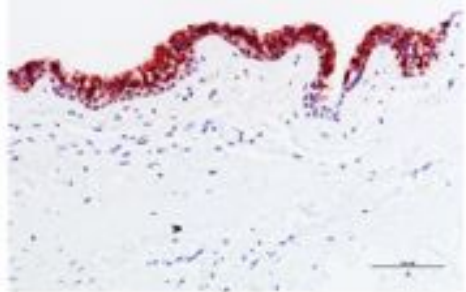

Alpha

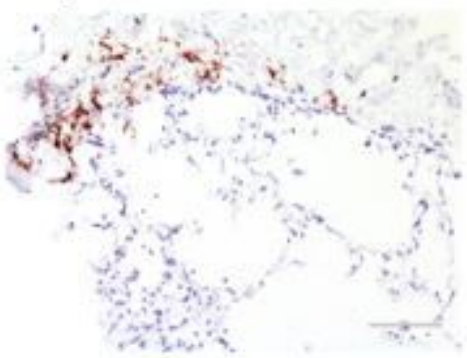

\section{Omicron}

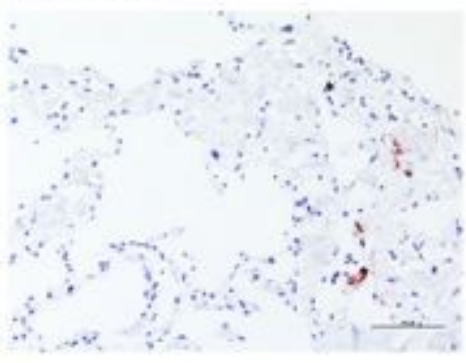

Figure 2 
Tissue tropism of SARS-CoV-2 variants in ex vivo cultures of human respiratory tract. Ex vivo cultures of

a. human bronchus and $b$. human lung were infected with various lineages of SARS-CoV-2 with $5 \times 10^{5}$ $\mathrm{TCID}_{50} / \mathrm{mL}$ at $37^{\circ} \mathrm{C}$ and the tissues were fixed with formalin at $72 \mathrm{hpi}$. Paraffin-embedded sections were subjected to immunohistochemical staining with a monoclonal antibody against the SARS-CoV-2 nucleoprotein. Positive cells are red-brown. Scale bar, $100 \mathrm{~mm}$. The images are representative of three individual donors. 

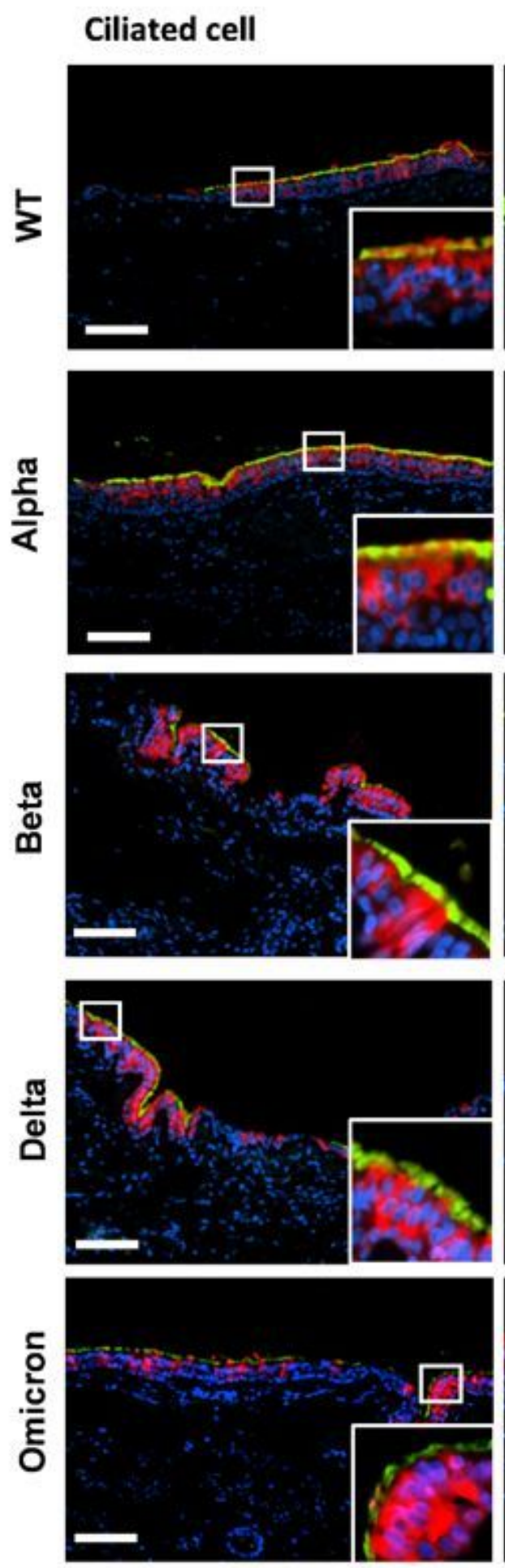

Goblet cell

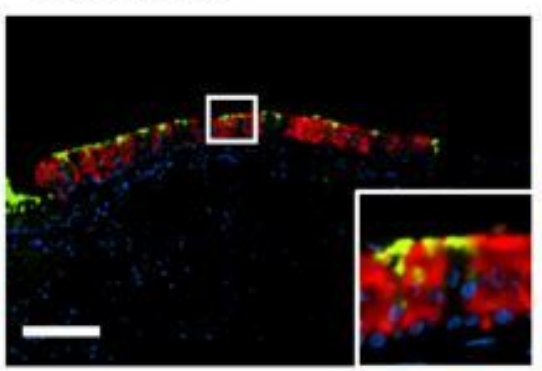

\section{Club cell}
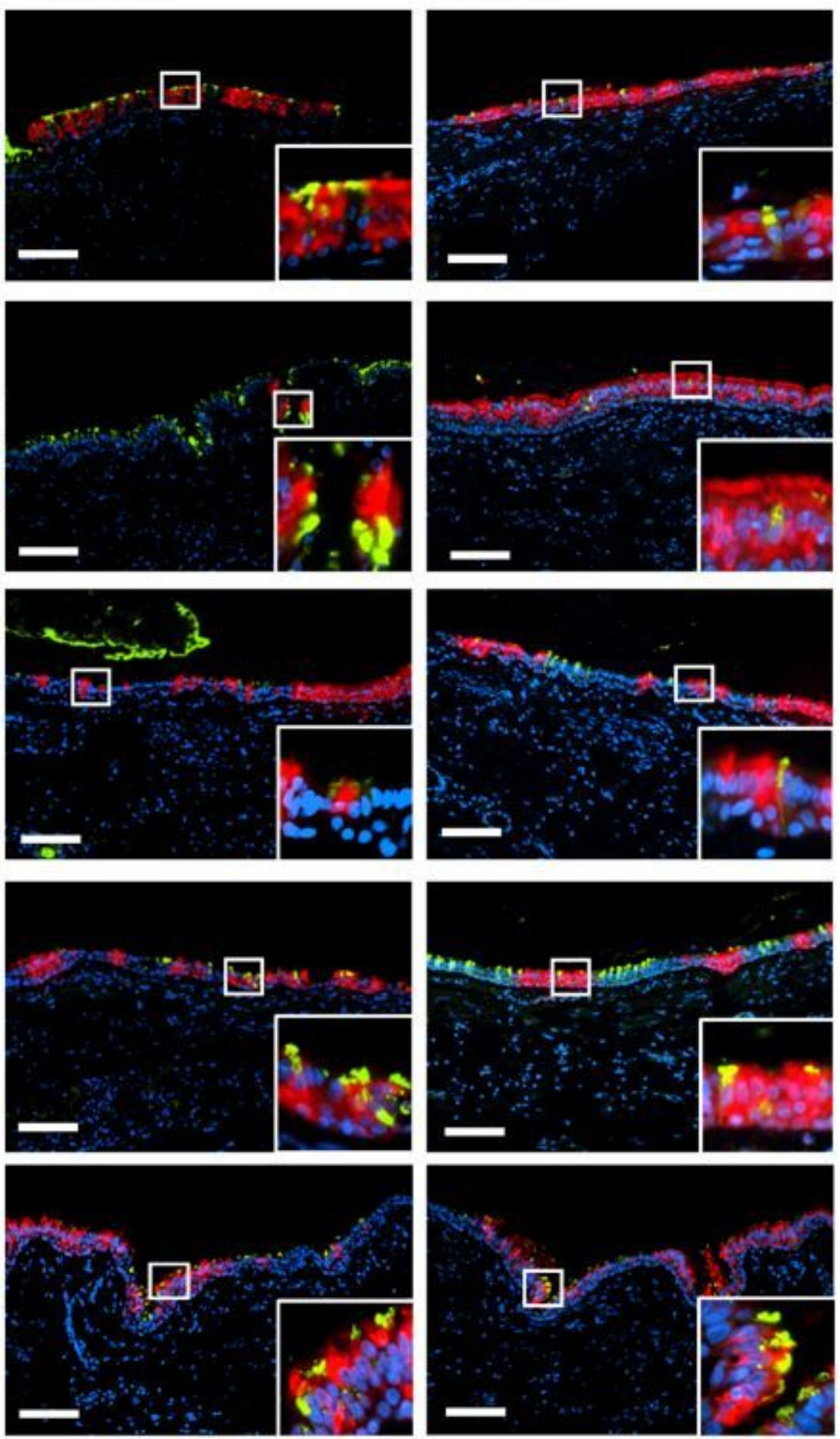

Figure 3

Cellular tropism of SARS-CoV-2 variants in ex vivo cultures of human bronchus. Ex vivo cultures of human bronchus were infected with SARS-CoV-2 wild-type (WT), Alpha, Beta, Delta and Omicron. At 72 hpi the tissues were fixed with formalin, embedded in paraffin and stained for immunofluorescence (green) for indicated cell markers: acetyl-a-tubulin-positive for ciliated cells, mucin 5AC-positive for secretory goblet cells and club cell protein 10-positive for club cells; a monoclonal antibody against the 
SARS-CoV-2 nucleoprotein (red) and 4',6-diamidino-2-phenylindole-positive for nuclei (blue). The images are representative of three individual donors. Scale bar, $100 \mathrm{~mm}$.

a

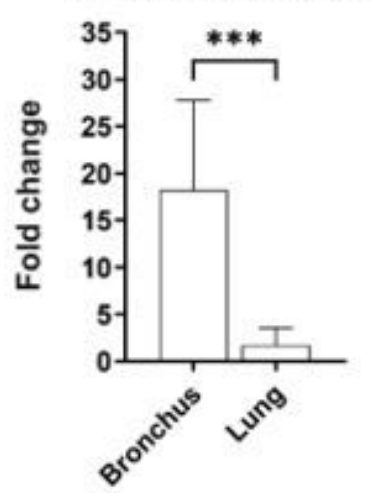

b ACE2-long and short

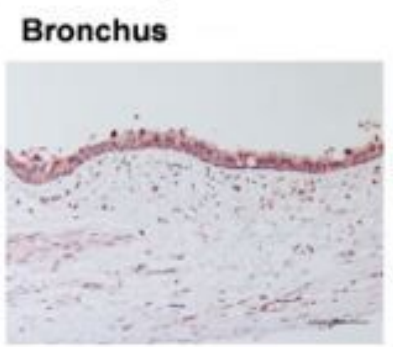

d
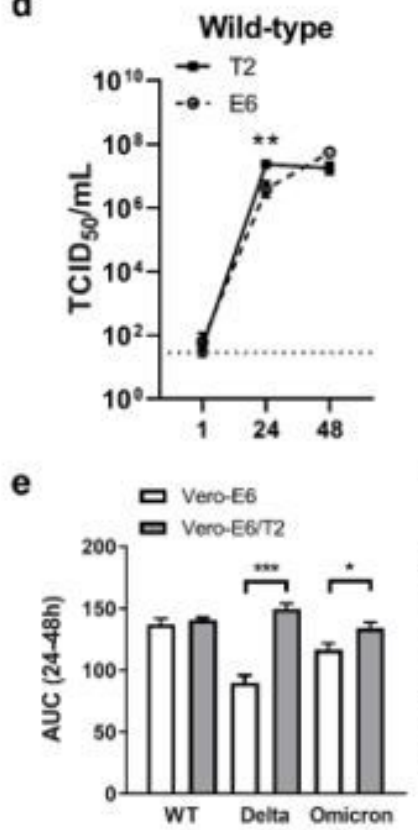

ACE2-short

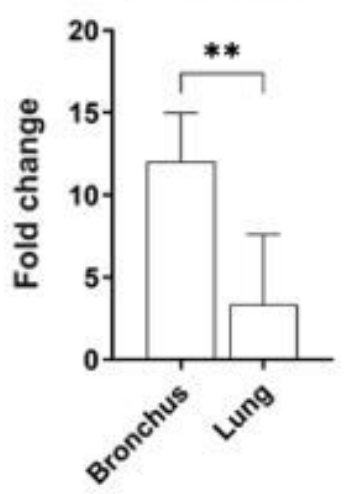

Lung
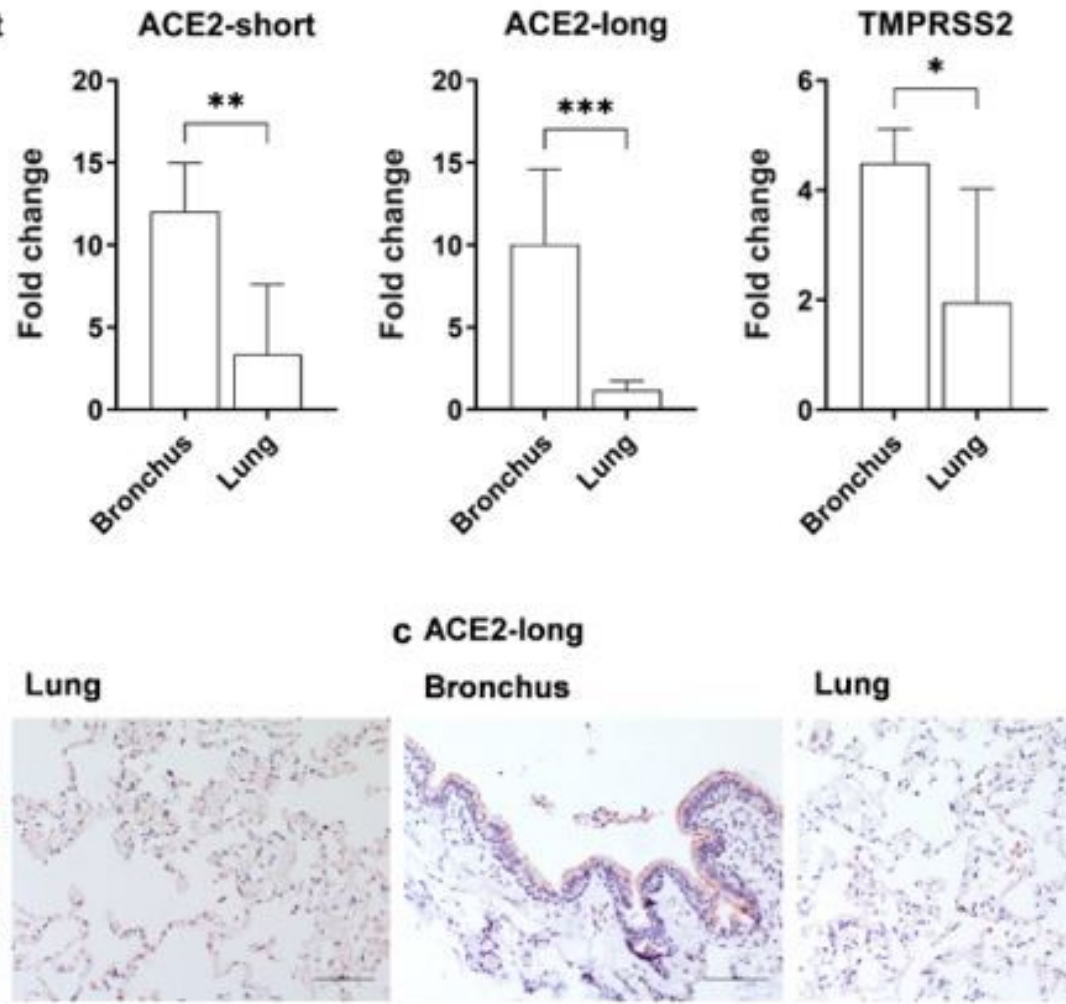

C ACE2-long

Bronchus

Lung
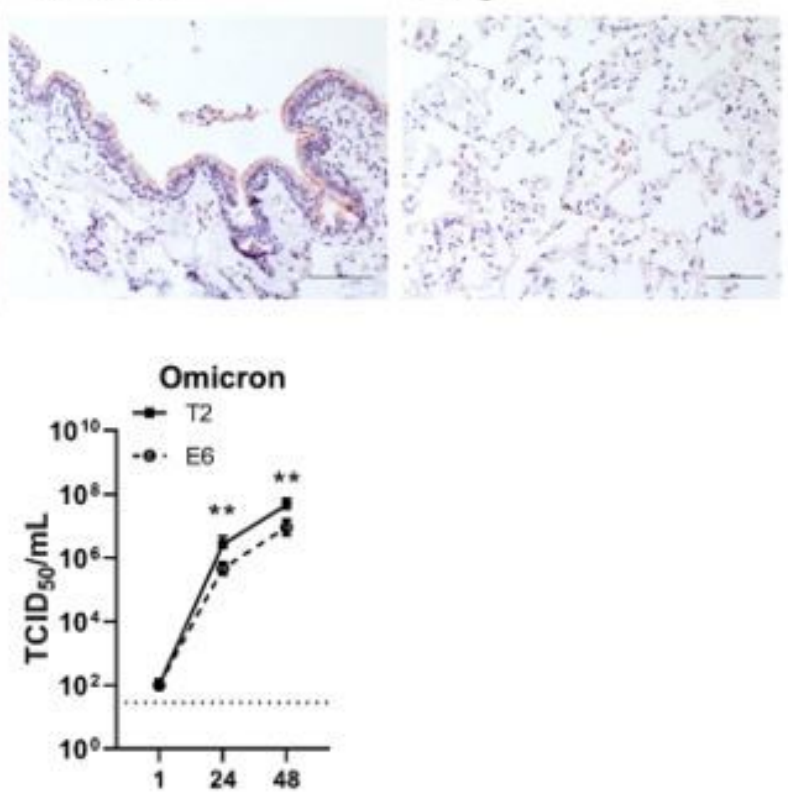

f

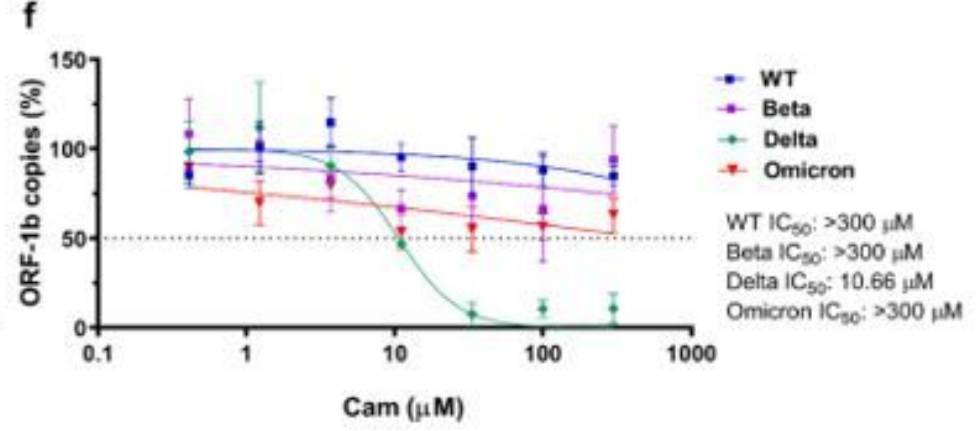

Figure 4 
ACE2 expression of respiratory tissues and TMPRSS2 dependence of SARS-CoV-2 variants. ACE2

expression in the human bronchus and lung tissues. a. RNA of human bronchus and lung tissues was extracted and the mRNA expression of non-specific (both long and short), short, long form of ACE2 and TMPRSS 2 was measured by real-time PCR. Graphs show mean fold change normalized with the gene expression in lung tissues. Data are mean of 3 (bronchus) to 4 (lung) individual donors. Statistics were performed using unpaired Student $t$ test. ${ }^{*} p<0.05$, ${ }^{*} p<0.01,{ }^{* \star} p<00.001$. b, c. Human bronchus and lung tissues were fixed with $10 \%$ formalin. Paraffin-embedded sections were subjected to immunohistochemical staining with antibodies distinguishing non-specific (both long and short) form and long form of ACE2. Positive cells are red-brown. Scale bar, $100 \mathrm{~mm}$. The images are representative of three individual donors. d-f. Viral replication kinetics of the variants in Vero E6, Vero E6-TMPRSS2 cells and the effects of treatment with camostat mesylate. d. Viral replication kinetics of Wild-type (WT), Delta and Omicron in Vero E6 (E6) and Vero E6-TMPRSS2 cells (T2). E6 and T2 cells were infected with SARS$\mathrm{CoV}-2$ viruses at $\mathrm{MOI} 0.1$. Viral loads in culture supernatants were harvested at the indicated times and virus titers were measured by $\operatorname{TCID}_{50}$ assay. Line-charts show the geometric mean virus titer of three independent experiments (SD). The horizontal dotted line denotes the limit of detection in the $\operatorname{TCID}_{50}$ assay. Statistics were performed using Two-way ANOVA followed by a Tukey's multiple-comparison test. ${ }^{* *} p<0.01, * * \star p<0.001$. e. Viral titers from $d$ are depicted as area under the curve (AUC). Bar-charts show the mean of three independent experiments (SD). Statistics were performed using unpaired Student $t$ test. ${ }^{*} p<0.05,{ }^{*} * p<0.001$. $\mathrm{f}$. Effects of viral replication by treatment with camostat mesylate (Cam). Vero E6TMPRSS2 cells were pretreated with Cam for $1 \mathrm{~h}$ before virus exposure. Infection of WT, Beta, Delta and Omicron was performed at $\mathrm{MOI} 0.05$ in the presence of the drug. After infection, the cells were replenished with fresh medium in the presence of Cam. Culture supernatants were collected at $48 \mathrm{hpi}$. ORF1b gene copies were measured by real time PCR. Line charts show the mean percentage of ORF1b gene copies using vehicle treatment as reference. Data are the mean of two independent experiments each with duplicates or triplicates $(n=5$ or 6$)(S D)$. The half-maximal inhibitory concentration $\left(I C_{50}\right)$ was indicated. 